\title{
Design of a Bio-inspired Dynamical Vertical Climbing Robot
}

\author{
Jonathan E. Clark ${ }^{1}$, Daniel I. Goldman ${ }^{2}$, Pei-Chun Lin ${ }^{1}$, Goran Lynch ${ }^{1}$, \\ Tao S. Chen ${ }^{3}$, Haldun Komsuoglu ${ }^{1}$, Robert J. Full ${ }^{3}$, and Daniel Koditschek ${ }^{1}$ \\ ${ }^{1}$ GRASP Laboratory, University of Pennsylvania \\ Department of Electrical and Systems Engineering \\ 200 S. 33rd Street, Philadelphia, PA, USA \\ ${ }^{2}$ School of Physics, Georgia Institute of Technology \\ ${ }^{3}$ PolyPedal Laboratory, Department of Integrative Biology \\ University of California at Berkeley, Berkeley, CA 94720, USA \\ email: jonclark@seas.upenn.edu
}

\begin{abstract}
This paper reviews a template for dynamical climbing originating in biology, explores its stability properties in a numerical model, and presents empirical data from a physical prototype as evidence of the feasibility of adapting the dynamics of the template to robot that runs vertically upward.

The recently proposed pendulous climbing model abstracts remarkable similarities in dynamic wall scaling behavior exhibited by radically different animal species. The present paper's first contribution summarizes a numerical study of this model to hypothesize that these animals' apparently wasteful commitments to lateral oscillations may be justified by a significant gain in the dynamical stability and, hence, the robustness of their resulting climbing capability.

The paper's second contribution documents the design and offers preliminary empirical data arising from a physical instantiation of this model. Notwithstanding the substantial differences between the proposed bio-inspired template and this physical manifestation, initial data suggest the mechanical climber may be capable of reproducing both the motions and ground reaction forces characteristic of dynamical climbing animals. Even without proper tuning, the robot's steady state trajectories manifest a substantial exchange of kinetic and potential energy, resulting in vertical speeds of $0.30 \mathrm{~m} / \mathrm{s}(0.75 \mathrm{bl} / \mathrm{s})$ and claiming its place as the first bio-inspired dynamical legged climbing platform.
\end{abstract}

\section{INTRODUCTION}

Past climbing robots have been slow and in most instances restricted to targeted surfaces where specific attachment mechanisms such as suction and electromagnetic adhesion can be brought to bear [1], [2]. Recently, robots have been built that are capable of more broadly effective attachment, for example by means of footholds [3, 4, 5] or vectored thrust [6, 7]. The last few years have also seen the revival [8], [9] of an older design [10] that used rimless wheels with sticky toes to intermittently 'roll' up smooth walls. To our best knowledge, no legged machine has climbed vertically in a dynamical manner, i.e., exploiting a controlled exchange of potential and kinetic energy in order to gain elevation.

The unremitting cost of work against gravity seems dramatically less constraining in the animal kingdom which boasts a variety of species that can dynamically speed their way up vertical environments surfaced in a broad variety of materials, textures, and geometries. Recent bio-mechanical studies of small, agile, climbing animals reveal a striking similarity in locomotion dynamics that belies stark differences in attachment mechanisms, morphology, and phylogeny [11]. These unexpectedly common patterns can be abstracted in a simple numerical model that raises the prospect of a "template" [12] for dynamical climbing analogous to the ubiquitous SpringLoaded Inverted Pendulum (SLIP) model [13, 14, 15] in sagittal level ground runners and Lateral-Leg Spring (LLS) [16] in sprawled level ground (horizontal) runners. In this paper we explore the value and applicability of this new biological climbing template to the domain of robotics. Specifically, we desire to build a fast, agile climbing robot capable of dynamical operation across a broad variety of scansorial regimes, and we wish to test the proposition that adapting this new biological template will prove both viable and effective to that end.

We present preliminary empirical evidence that such a robot may be fast and hypothesize about why it may prove agile as well. Specifically, we review the dynamical model of interest, introduce the design of a simple physical instantiation, and describe its early implementation in an account organized as follows: Section II reviews the template model, describing its origins in animal studies and exploring the effects of altering the leg sprawl (and therefore its lateral inpulling foot forces) on the speed and stability of climbing. Section III begins with a discussion of how the template can be scaled to our target robot mass, and evaluated the consequent power requirements. We next introduce a design for a simple legged climbing platform that reconciles the limitations of off-the shelf actuators with the power demands of the scaled model [17]. Section IV describes a physical implementation of this very simple template-inspired climber and compares initial data taken from early climbing experiments to the simulation studies. We conclude by commenting on some of the broader issues associated with robot climbers, and discuss future work including limb coupling dynamics, energetics, and adaptation to a more utilitarian polypedal morphology. 


\section{Template AND Stability}

Organisms as diverse as arthropods and vertebrates use differing limb number, attachment mechanism and body morphology to achieve performance on vertical substrates that rivals level ground running. Therefore, we expected that diverse animals would necessarily use different climbing strategies. In previous work [11] we have discovered common dynamics in quite different rapidly climbing organisms, a cockroach and a gecko. Surprisingly, neither climbs straight up a vertical flat wall. Both organisms generate substantial lateral forces during climbs over 4 bodylengths per second that produce substantial changes in lateral as well as fore-aft velocity [11, 18].

Significantly, the resultant ground reaction forces generated by these animals while climbing are distinct from the forces that the organisms use on the ground. The lateral forces are of opposite sign. On the ground both cockroaches and geckos limbs push away from their midlines, while on the level they pull toward the midline $[11,19,18]$.
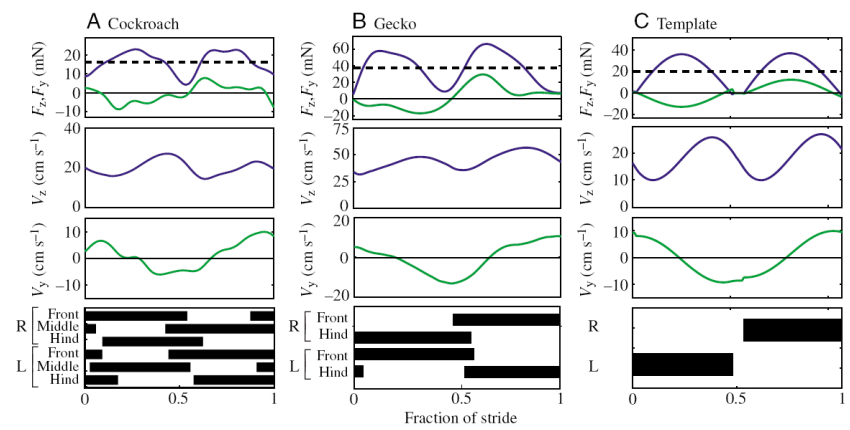

Fig. 1. Force, vertical velocity, lateral velocity, and foot fall patterns for the cockroach, gecko, and template. Broken lines indicate body weight. Data are shown for a normalized stride, with black bars representing foot contact. In each force plot $F_{z}$ is the magnitude in the vertical direction and $F_{y}$ is in lateral direction. Reproduced with permission from [11]

\section{A. Template Description}

A simple model which generates the template dynamics of vertical climbing is shown in Fig. 2a and a schematic of its motion in Fig. 2b. The model consists of a rigid body that is pulled upward and side-to-side through the action of a spring in series with a linear actuator.

As shown in Fig. 2, in the first step with the right leg, at touchdown $(t=0)$ the right actuator is maximally extended, and the spring is un-extended with zero rest length. Touchdown is created by establishment of a rotationally free pin joint with the wall. As the actuator length $L(t)$ decreases, the spring in the leg extends, the foot freely pivots about the point of contact and the center of mass $(\mathrm{COM})$ is translated vertically and laterally. The cycle repeats for the left leg. The actuator changes length sinusoidally such that $L(t)=$ $L_{0}(1+z \sin (2 \pi f t))$, where $z$ is the fractional length change, $\mathrm{f}$ is the stride frequency. The solid vertical line in each panel indicates the fixed lateral position about which the center of mass laterally oscillates. The angular excursion of the body and extension of the spring are exaggerated for clarity. Actual angular excursion of the body relative to vertical is approximately \pm 3 degrees. The model was coded and integrated in the Working Model 2D (Design Simulation Technologies, Inc) simulation environment. The parameters used to generate Fig. $1 \mathrm{C}$ were body mass $=2 \mathrm{~g}$, body dimensions $=4 \mathrm{~cm} \times 0.95$ $\mathrm{cm}, l_{1}=0.71 \mathrm{~cm}, l_{2}=0.84 \mathrm{~cm}, \beta=10$ degrees, $L_{0}=1.54$ $\mathrm{cm}, z=0.6, k=6 N m^{-1}, \gamma=0.09 N-s m^{1}, f=9 \mathrm{~Hz}$. The attachment duty factor in the model is 0.46 . The rigid body has a moment of inertia of $8 \times 10^{-7} \mathrm{~kg}-\mathrm{m}^{2}$, the order of magnitude of cockroaches $\left(2 \times 10^{-7} \mathrm{~kg}-\mathrm{m}^{2}\right)$ [20].

The forces and resulting center of mass velocities generated by the model are shown in Fig. 1 and agree well with the pattern measured in cockroaches and the geckos.

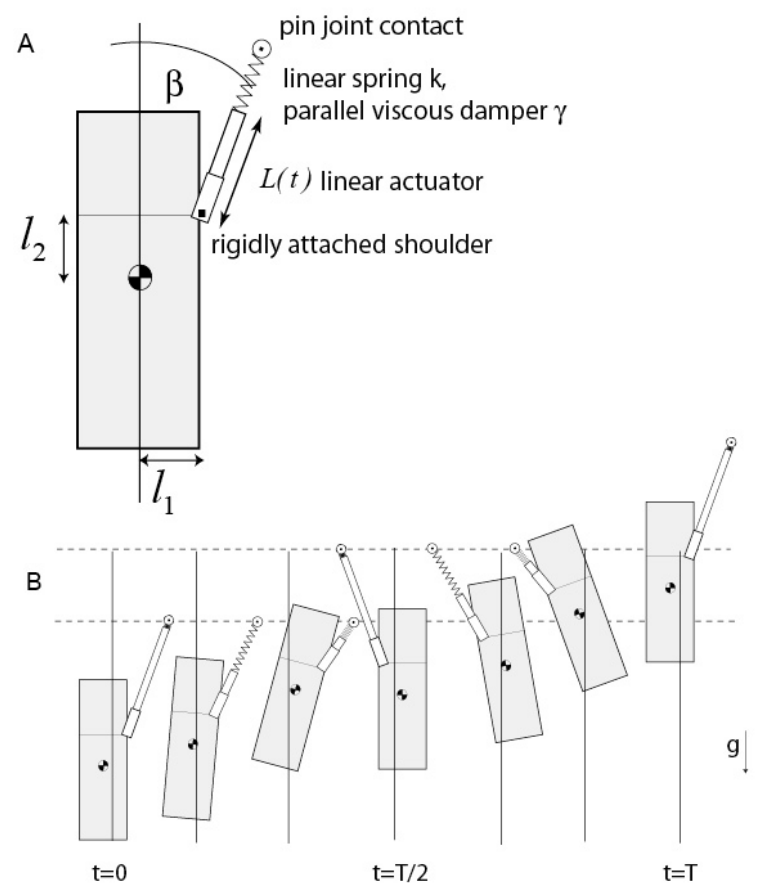

Fig. 2. A dynamic template for climbing. The two degree of freedom model that generates the template climbing dynamics shown in Fig. 1C. (A) Schematic of the model. (B) Schematic of the motion of the model during two steps. Reproduced with permission from [11].

\section{B. Lateral Force and Sprawl Angle}

One of the most intriguing findings from the study of vertically running animals is the presence of large lateral velocities and in-pulling forces. At first blush these seem to be wasteful, since substantial muscular force is developed in a direction orthogonal to the climbing direction. One possible explanation for their presence is that generation of lateral forces is necessary for proper adhesion at the feet for both the dry adhesive toe pads of geckos and the pads and claws of cockroaches. Another possible reason is that the addition of lateral dynamics aids stability. In the template model the lateral motions and forces are due to the alternating pendular nature of 
the climbing dynamics. To what extent does the effective splay or sprawl angle of the legs of the template affect the motion and stability of the climber? Is their a dynamic benefit to having large lateral forces? To begin to answer these questions we fix the dimensions of the template, parameters $k, \gamma$, and touchdown timing, but vary the sprawl angle $\beta$ (Fig. 2).

The dynamics generated are shown in Fig. 3. As $\beta$ increases, the speed reaches a maximum (Fig. $3 b$ ) near $\beta \approx 30$ degrees, then decreases as the template no longer is generating enough fore-aft force to pull against gravity, but instead produces only lateral forces (for $\beta=90$, the climber cannot ascend).
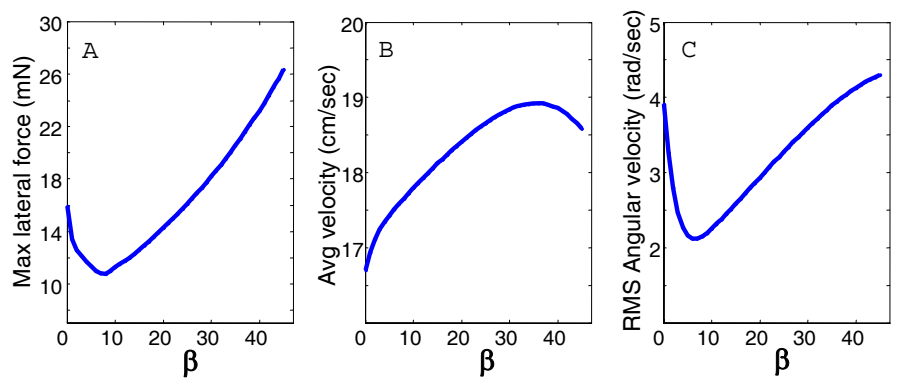

Fig. 3. Variation of template dynamics with as $\beta$, the angle of the leg, increases from 0 to 45 degrees. (a) Lateral force (b) Average climbing velocity. (c) Angular velocity.

Surprisingly, we find that as $\beta$ changes, the template's angular velocity and peak lateral force do not increase monotonically, but instead display minimum around $\beta \approx 10$, see Fig. 3a,c. Furthermore, this minimum corresponds to the approximate effective angle or force ratio that the organisms use to climb, see Fig. 4.

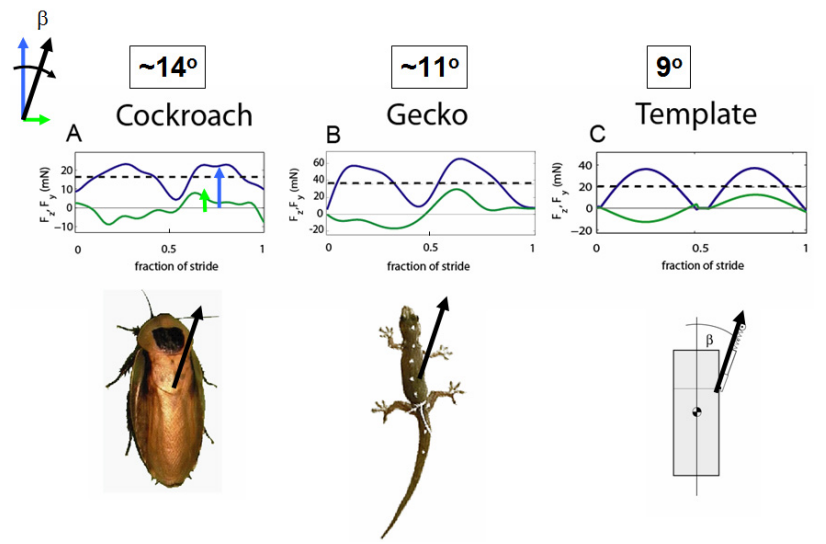

Fig. 4. The $\beta$, or effective leg angle, used by the many-legged organisms during vertical climbing is similar to that selected to match COM dynamics.

\section{Perturbations}

A preliminary investigation of the template response to perturbation reveals hints as to why large lateral forces may

be advantageous in climbing. We note that for an organism (or template) of length $l$ the pendular frequency associated with a single limb in contact is on the order of $1 / 2 \pi \sqrt{g / l}$. For an organism or template of length of $5 \mathrm{~cm}$, this is about $3 \mathrm{~Hz}$. Since the animals climb with stride frequency on the order of $10 \mathrm{~Hz}$, this slow pendular oscillation has potential to dominate the dynamics and must be either actively or passively controlled.

In Fig. 5, we demonstrate that generation of lateral forces while climbing results in passive self-stability to the low frequency pendulum oscillation. Upon a lateral impulsive perturbation of about $2 \mathrm{mNs}$, the climber that only pulls straight up the wall is still swinging wildly 20 steps $(1 \mathrm{sec})$ later. The template that generates lateral forces with $\beta \approx$ 10 has corrected for the perturbation within a few strides. This correction does not result from active sensing of the perturbation, but instead emerges from the dynamics.
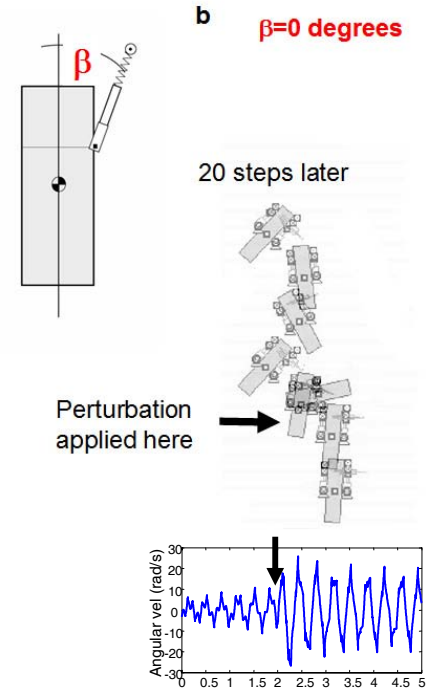

b $\quad \beta=0$ degrees

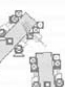

Fig. 5. (a) Template with $\beta=10$ degrees recovers rapidly after perturbation. Template that pull directly above $(\beta=0)$ swings out of control and never recovers.

The effect of leg angle $(\beta)$ on the response to perturbation is summarized in Fig. 6. Here we see that 1.5 second (20 steps) after perturbation, the dynamics for $\beta<10$ still differ from before the perturbation, but for $\beta>10$, the dynamics have returned to the natural gait.

Thus it appears that a $\beta=10$ is about the smallest leg sprawl angle for the pendulous template climber that will automatically reject large perturbations quickly. Operation at this posture also results in a minimum RMS angular velocity and the lowest lateral forces (as shown in Fig. 3). We suspect that it is not accidental that this is the same effective $\beta$ where the ratio lateral/vertical wall reaction forces matches those found in the dynamic animal climbers, as shown in Fig. 4.

\section{Scaling The Template}

With a biologically inspired template in hand, the first step in constructing a robot based on this model was to determine 

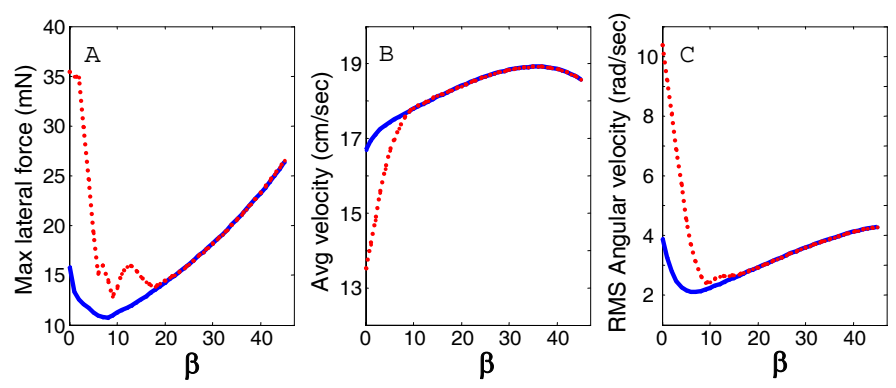

Fig. 6. The effect of $\beta$ on the recovery from a lateral perturbation with impulse on the order of $2 \mathrm{mN}$-sec. (a) Lateral force (b) Average climbing velocity. (c) Angular velocity. In each plot the solid line is the steady-state behavior (before impulse) and the dotted line is the state 1.5 seconds after impulse.

the appropriate scale for construction. As a target we chose the size and power-density of RiSE $[5,21]$ — the first general purpose legged machine to exhibit reliable climbing behavior on a variety of natural and synthetic surfaces. In order to preserve the motions and stability results for the template, the scaled model should preserve dynamic similarity.

\section{A. Scaling Rules}

Geometric scaling is preserved by changing all length by the same amount. Stiffness, mass, damping, and frequencies, however need to change by different ratios to ensure dynamic similarity. If dynamic similarity is preserved then all of the resulting displacements, times, and forces are scaled versions of the original [22], and the stability characteristics are invariant [20].A more detailed discussion of dynamic similarity and a derivation of the associated scaling laws adapted here is given in [23]. The necessary transformations are summarized in table I.

TABLE I

DYNAMIC Similarity SCALING FACTORS

\begin{tabular}{l|c|c|c|c}
\hline Quantity & $\alpha_{x}$ & Relation & $f\left(\alpha_{L}\right)$ & $\alpha_{L}=10$ \\
\hline Mass & $\alpha_{m}$ & & $\alpha_{L}^{3}$ & 1000 \\
Frequency & $\alpha_{\omega}$ & $\sqrt{\frac{1}{\alpha_{L}}}$ & $\alpha_{L}^{-1 / 2}$ & 0.316 \\
Stiffness & $\alpha_{k}$ & $\alpha_{\omega}^{2} \alpha_{L}^{3}$ & $\alpha_{L}^{3}$ & 100 \\
Velocity & $\alpha_{v}$ & $\alpha_{\omega} \alpha_{L}$ & $\alpha_{L}^{1 / 2}$ & 3.16 \\
Damping & $\alpha_{b}$ & $\frac{\alpha_{L}^{3}}{\alpha_{v}}$ & $\alpha_{L}^{5 / 2}$ & 316 \\
Power & $\alpha_{P}$ & $\alpha_{L}^{3} \alpha_{v}$ & $\alpha_{L}^{7 / 2}$ & 3160 \\
\hline
\end{tabular}

Each term has a scaling factor $\left(\alpha_{x}=x_{2} / x_{1}\right)$ where $\left(x_{2}\right)$ is the scaled quantity and $\left(x_{1}\right)$ is the original. The last column shows how much the various quantities need to change for a length scale $\left(\alpha_{L}\right)$ increase of 10 (i.e. the change between a $2 \mathrm{~g}$ cockroach and a $2 \mathrm{~kg}$ robot).

When we increase the length $\left(\alpha_{l}\right)$ by a factor of 10 , we also increase the needed power to weight ratio by:

$$
\frac{\Delta \text { power }}{\Delta \text { weight }}=\frac{\alpha_{P}}{\alpha_{m}}=3.16
$$

Since the power density of actuators is relatively mass independent, it becomes more difficult to provide the power necessary to achieve the template-based dynamics as our scale increases.

The historically achievable power density of climbing and running robots varies greatly, but as a point of reference both the hexapedal RiSE and RHex [24] robots have a specific power of approximately $10 \mathrm{~W} / \mathrm{kg}$ per tripod. The template model, at the cockroach scale, requires a peak of $6.3 \mathrm{~W} / \mathrm{kg}$ per leg. However, scaling to a $2 \mathrm{~kg}$ climber increases this power demand by 3.16 to about $20 \mathrm{~W} / \mathrm{kg}$ per leg. Thus the model requires the availability of twice as much peak power from the motors as has been available in these previous robotic designs.

\section{B. Design modifications}

A numerical study [17] investigating how to reconcile the template's power requirements with the considerably reduced power densities to be found in high performance commercialoff-the-shelf electromechanical servo motors suggested a recourse to three independent design modifications.

The first proposed modification substitutes a DC motor model and crank slider mechanism to replace the prismatic actuators of the template. The second introduces a passive-elastic element in parallel with the leg actuator to store energy during the swing-recirculation phase of the leg motion, and then to release the energy during stance to aid with accelerating the body upwards. The third modification substitutes a force-based rather than position-based control scheme for the legs. Fig. 7 depicts these changes.

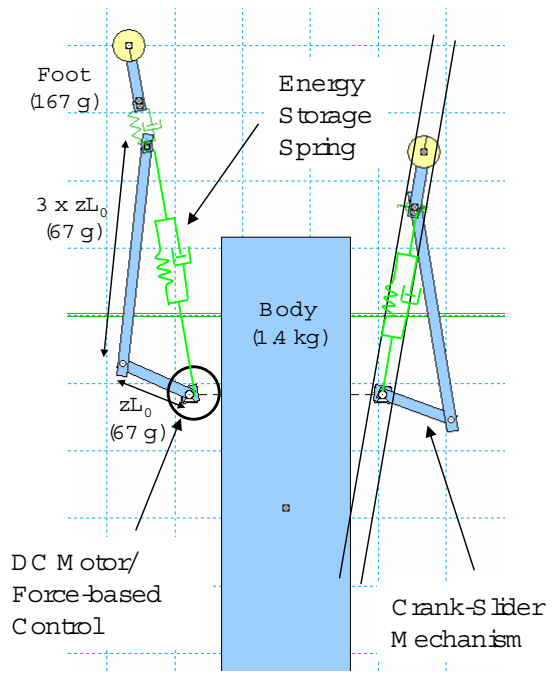

Fig. 7. Schematic of the crank-slider mechanism used to convert rotary (motor) output into linear motion. The relative lengths and masses of the links are indicated.

With the the altered kinematics and additional leg springs $(\mathrm{k}=130 \mathrm{~N} / \mathrm{m}, \mathrm{b}=3 \mathrm{~N}-\mathrm{s} / \mathrm{m})$ we found [17] that the peak power 
required for each legs dropped from $40 \mathrm{~W}$ to a more reasonable $25 \mathrm{~W}$ during steady state climbing.

\section{Results with force-maximizing control}

The consequences of these combined modifications in the numerical model are detailed in [17] and summarized in Fig. 8.
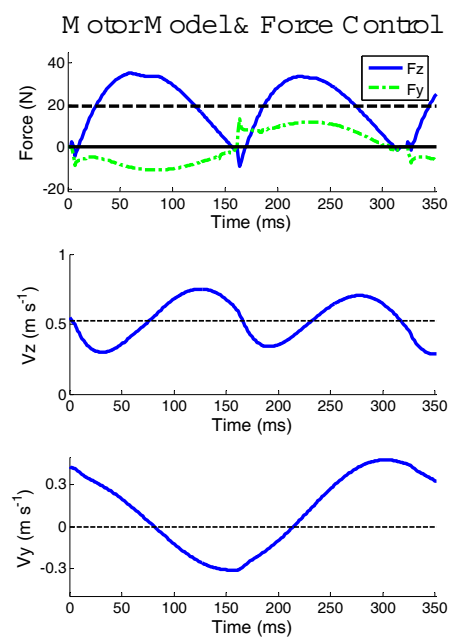

Fig. 8. Force, vertical velocity, and lateral velocity for proposed modified template climber with a motor model and force-based control scheme.

A comparison of the dynamics reveals that the modified model is, despite the scaling, essentially the same as the original template. The switch from a trajectory-tracking to a force-based control scheme releases our control of the resulting actuation frequency. While this frequency shifting during climbing can increase the performance of robot, it also complicates the dynamic coupling between the leg switching, body rotation, and the wrist-spring extension. While this could alter the motion and efficiency of the model, the simulation results suggest that for the motor model chosen the resulting steady-state trajectories work well. In any case, the transients of the dynamics are an additional factor to consider when designing the controller.

The net result, however, is a realistically sized and powered dynamic climber that is very close to the template derived from animal studies. The overall projected climbing speed of 0.55 $\mathrm{m} / \mathrm{s}$ compares very favorably to that of the scaled template $(0.60 \mathrm{~m} / \mathrm{s})$.

\section{RoBOt DESIGN AND EXPERIMENTS}

The general design philosophy guiding the development of this initial physical model was to investigate the efficacy of the proposed template with respect to realistically available power density. To maintain this focus, we sought to decouple the vertical and lateral climbing dynamics from other key environmental interactions required for climbing such as adhesion to the wall. Initially, we considered using electromagnets or a specially prepared surface to ensure that each foot hold was secure while climbing. While such "cheating" with respect to foot attachment mechanics would have been viable, we feared that it would unduly hinder our eventual goal of integration of this physical climbing model into a versatile climbing robot. We chose instead to work with claw-like feet on a carpet substrate, a combination that proved effective as a starting point for RiSE [5], and on which that robot's fastest climbs have been recorded [25]. This initial setting gives us confidence that the attachment developments that have enabled RiSE to move from carpet to brick, stucco, concrete, etc. [21] might be adaptable to our climbing model as well. It also provides for an equitable comparison of the robots' relative performance.

\section{A. Mechanical Structure and Design}

The basic mechanical design is adapted directly from the two-dimensional simulation presented in [17], which in principle is comprised of a rigid body and two linear-moving hands with springs. The resulting robot, depicted in Fig. 9, features two motors, each driving a crank-slider mechanism attached to an arm. As in simulation, each leg has a energy-storage spring in parallel to the crank-slider mechanism with a stiffness and set point designed to assist the leg while retracting during stance. Each foot also features a pair of passive-wrist springs which act in series with the drive actuation. These passively connect the hand to arm and are extended during the beginning of the stance phase, acting to mitigate the loading forces on the robot. Heavy components like motors are located below the cranks in order to shift the location of center of mass lower to match configuration in the template. The frame of robot is constructed from ABS plastic, and the transmission system is mainly comprised of a pair of bevel gears, a pulley pair, sliders (steel shafts and linear bearings), and aluminum links. The sprawl angles $\beta$ of both arms are adjustable with several pre-settings, including the nominal setting of $\beta=10$.

We implemented a bar across the rear of the robot extending laterally $20 \mathrm{~cm}$ on both sides to reduce the roll of the robot. This bar approximates the function of multiple legs in reducing the dynamics to the planar case considered in the template. The robot's physical parameters are summarized in table II.

TABLE II

Physical PARAMETERS FOR THE ROBOT

\begin{tabular}{|l|l|}
\hline Body size & $400 \times 116 \times 70 \mathrm{~mm}$ (excluding cables) \\
Body mass & $2.4 \mathrm{~kg}$ \\
Effective Stride frequency & $1.9 \mathrm{~Hz}$ \\
\hline Wrist Spring Stiffness & $640 \mathrm{~N} / \mathrm{m}$ \\
Arm Spring Stiffness & $140 \mathrm{~N} / \mathrm{m}$ \\
Motor & Maxon RE 25118752 \\
& Power: 20 watts \\
& Size: $\phi 25 \mathrm{~mm}$ \\
& Nominal voltage: $24 \mathrm{~V}$ \\
& No load speed: $9660 \mathrm{rpm}$ \\
& Maximum torque: $240 \mathrm{mNm}$ \\
& Maximum current: $1230 \mathrm{~mA}$ \\
Gear head & Maxon Planetary Gearhead GP 32A 114473 \\
& $33: 1$ Gear ratio \\
Encoder & Maxon digital encoder HEDS55 110515 \\
& 500 count/turn \\
Bevel Gear & $2: 1$ reduction \\
Leg stroke & 120 mm \\
\hline
\end{tabular}




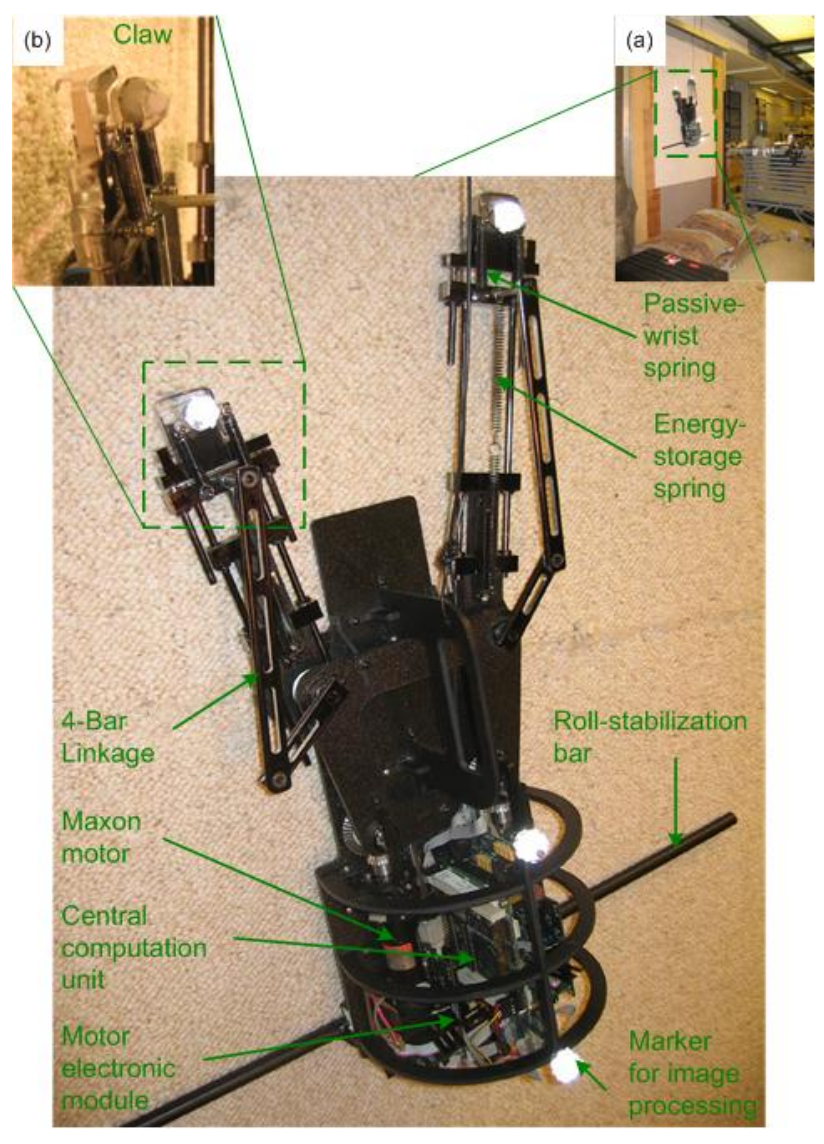

Fig. 9. Picture of the robot with annotations.

We chose to implement a passive attachment strategy where the claw is rigidly attached to the hand. The bent shape highlighted in the blow-up (b) in Fig. 9 engages the substrate when the leg is being pulled down, and releases when pushed. A slight pitch angle introduced by a block under the tail of the robot ensures that the extended foot is closer to the wall than the retracted foot and aids in attachment. Limitations in this technique, however, result in occasionally lost footholds, and a (sometimes significantly) shortened stride length.

Future foot designs include an actuator at the hand which will improve the reliability of attachment, and provide for control of the phasing of attachment and detachment.

\section{B. Electronics and Software}

Our robot employs a family of electronic and software components designed for building modular and extensible mechatronic systems governed by distributed control architectures. These components are members of a larger general purpose robot building infrastructure that consists of electronic and software building blocks and standardized interfaces facilitating easy composition.

The present implementation uses three components: 1) a Linux-based $366 \mathrm{MHz}$ CPU card; 2) a carrier board; and 3) two 1-DOF motor controllers. The carrier board interfaces the CPU board with all of the peripheral components. High level control commands are executed on the CPU board, with local feedback control occurring at the motor controllers.

The high level control software is written using RHexLib - a software library which has been developed and used for other legged robots [24], [5] to implement time trigger architecture (TTA) digital control software. This code base provides a library of functions as well as a GUI.

The modular nature of this implementation will become more important in the later stages of this study as we incorporate additional actuators and sensors.

\section{Controller}

In contrast to the RiSE and RHex machines whose initial leg coordination obtains from a purely feedforward centralized "clock," the alternating leg oscillations arise in the present model from two self-stabilizing mechanical limit cycles that are coupled through the mechanics of the leg-body-ground interactions - a purely decentralized feedback paradigm [26, 27]. Numerical exploration of these oscillators suggests that in their natural state of mutual coupling they stabilize around an undesirable in-phase synchronized gait.

Guided by early numerical studies and general principles from past work [27, 28, 29], we use a 4-state hybrid controller to prevent synchronization of the legs while maximizing the power driving the stance leg at all times.

Imposing de-synchronizing influences on these decentralized feedback driven hybrid oscillators can be achieved in an intuitively appealing manner by recourse to the notion of a "mirror law" first introduced in [30]. A leg in stance mode is commanded the highest permissible torque $\left(T_{\max }\right)$, while the leg in flight mode is controlled to follow the stance leg with an offset of $\pi$. Specifically:

$$
\begin{gathered}
T_{\text {stance }}=T_{\max } \\
T_{\text {flight }}=k_{p} *\left(\bmod \left(\theta_{f}-\theta_{s}-\frac{\pi}{2}, 2 \pi\right)-\pi\right)+k_{d} *\left(\dot{\theta}_{f}-\dot{\theta}_{s}\right)
\end{gathered}
$$

where $k_{p}$ and $k_{d}$ are controller gains and $\theta_{f}$ is the position of the leg in flight mode, and $\theta_{s}$ is for stance. Ideally, the robot would have both legs transition their states simultaneously, resulting in a situation in which one leg is always in flight mode and the other always in stance mode. However, frequently (and, indeed, systematically), both legs are in the same mode at the same time. In these cases, the controller continues to advance the leg which is closer to transitioning to a new state, while temporarily halting the other leg's progress. Though clearly sub-optimal, this algorithm empirically demonstrates convergence of the robot's gait to a stable limit cycle.

\section{Experimental Setup and Procedure}

To evaluate the robot climbing performance, a $2 \mathrm{~m} \times 0.8 \mathrm{~m}$ carpet-surface vertical climbing wall was built as shown in section detail (a) of Fig. 9. A commercial 6-axis force sensor (AMTI HE6x6) is installed on the wall to collect interacting forces between the left foot and the wall. A vision system composed by a commercial HD video camera (SONY HDRSR1) and two spotlights for robot motion traction is located 
$2 \mathrm{~m}$ away facing climbing wall. In order to simplify the offline analysis of the visual data, the robot is "blackened" and 4 spherical markers coated with reflective tape (3M) are installed: two on the body for size calibration and one on each hand for hand position.

Both force data and video data are collect while the robot climbs. Video streams are exported into sequential images for post processing in Matlab. Each color image is converted to black and white by setting threshold empirically and the "white" objects in the image are distinguished from each other by a labeling function and by their geometrical relations.

\section{E. Climbing Results}

Despite some significant limitations in this early physical instantiation, the robot climbs remarkably well. Figure 10 shows the trajectory and velocity of the center of mass of the robot while climbing.

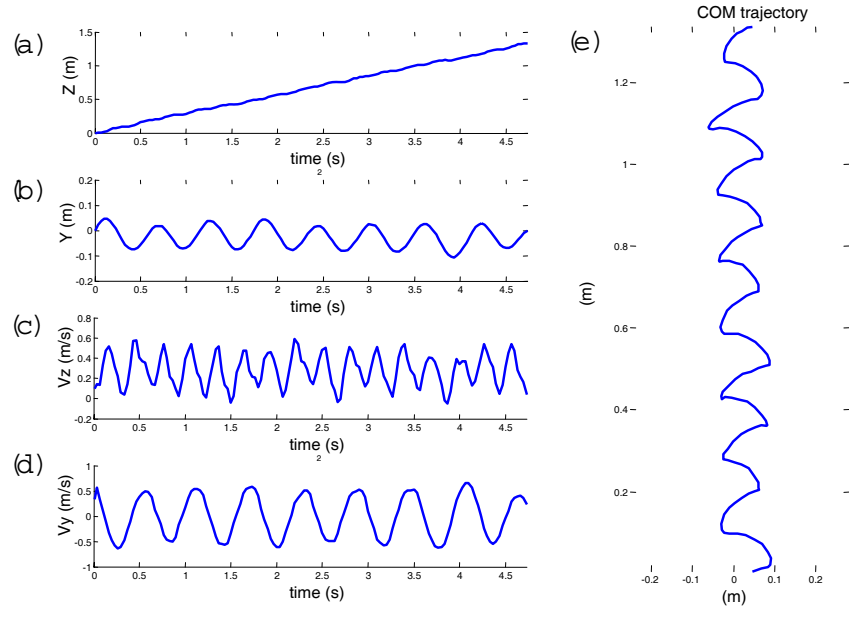

Fig. 10. Video-based marker data for six strides of the robot climbing. (a) vertical displacement, (b) lateral displacement, (c)vertical velocity, (d) lateral velocity, and (e) the path of the center of mass during climbing.

The COM position plot (e) shows that despite the limitations from attachment, convergent and repeatable gaits emerge. The magnitude of the lateral velocity is about 1.5 as large as than the simulation predicts, and the robot climbs at about $30 \mathrm{~cm} / \mathrm{s}$.

The physical climber is clearly not as effective as the simulation or the animals, but it does demonstrate that dynamic vertical climbing is in fact possible. Figure 11 compares the ground reaction forces and velocities of the robot and a scaled version of the template.

The magnitude of the vertical oscillations and velocity are considerably lower than expected. This is due to two factors: (1) we are operating at a conservative gear ratio, and (2) the effective stroke length due to attachment issues is reduced. With these issues resolved we believe that we should be able to significantly increase our effective climbing velocity.

For comparison the results of the $2 \mathrm{D}$ simulation and the robot are summarized in Table III.

Due to early power limitation issues, we utilized a conservativegear reduction (66 vs 50 ). This enabled us to climb,
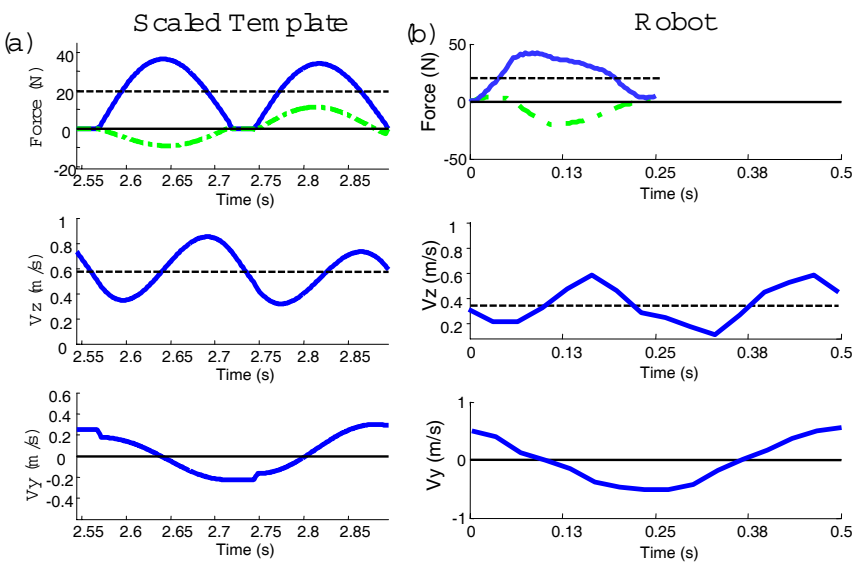

Fig. 11. Ground reaction forces and COM velocities for template and robot.

TABLE III

MOdel-Robot PerformanCE COMPARISON

\begin{tabular}{|c|c|c|}
\hline & Simulation (2kg) & Robot $(2.4 \mathrm{~kg})$ \\
\hline Peak Fz & $38 \mathrm{~N}$ & $46 \mathrm{~N}$ \\
Peak Fy & $11 \mathrm{~N}$ & $22 \mathrm{~N}$ \\
Stroke Length & $12 \mathrm{~cm}$ & $8.5 \mathrm{~cm}$ \\
Stride Freq & $2.5 \mathrm{~Hz}$ & $1.9 \mathrm{~Hz}$ \\
Velocity & $55 \mathrm{~cm} / \mathrm{s}$ & $30 \mathrm{~cm} / \mathrm{s}$ \\
\hline
\end{tabular}

but reduced the peak operational speed by $25 \%$. This in turn disturbs the balance between vertical extension of the leg and the rotation of the body-resulting in wider swings than predicted.

More pernicious in terms of upward velocity, however, is the effect of attachment on our effective stride length. The nominal length extension for each arm is $12 \mathrm{~cm}$, yet we are currently only seeing an effective stroke length for each step of $8.5 \mathrm{~cm}$. The improper passive attachment and detachment of the limbs is largely responsible for this discrepancy.

Despite these differences we still see a remarkable similarity between the robot and the model's wall reaction forces and in the phasing between the center of mass velocity and the footreaction forces, as shown in Fig. 11.

The result is that we are ill-tuned, but still climbing in a dynamic manner. Ongoing work to add active foot attachment, alter the gearing, and improve the control scheme should result in a much better match with the template, and faster and more reliable climbing.

\section{CONCLUSion AND Future Work}

A fundamental discovery of a common dynamical pattern, we term a template, for climbing in diverse animal species inspired the design of, first a simulation [17, 11], and now in he present study, a novel bipedal dynamic climber. A simulation of the template and initial experimental results from the robot testing show a remarkable correspondence. The addition of a force-assist spring in parallel with the actuator in the legs and the switch to a force maximizing control scheme allow for a dynamic climber to climb at slightly more than our target mass 
of $2 \mathrm{~kg}$. In addition, it appears that some of the characteristic force and motion patterns of the animals and the steady gaits exhibited by the template are reproducible in the physically anchored version, the robot.

Future theoretical work is required in the development of a robust leg synchronization scheme and in understanding how the structure and control scheme of the robot contribute to its lateral stability. Future experimental work will need to be carried out that includes investigating the effect of increased climbing speed on the attachment and detachment of feet, developing methods to deal with foot slippage or failed attachment, and integrating with RiSE into a dynamic climbing robot that can operate on a number of substrates and in a variety of situations.

Additional experiments will also be needed to support our hypothesis and simulation results that lateral and rotational stability for climbers is improved by generation of large steady-state lateral inpulling forces. For the robot this entails showing whether in fact utilizing a sprawl angle $(\beta)>10^{\circ}$ improves stability while climbing.

In conclusion, we have built the first, bio-inspired, dynamical climbing robot. The successful scaling and implementation of the bio-inspired template has enabled us to explore the possible advantages of this novel design that uses passivedynamic elements. Because the robot has the same dynamics measured in diverse animal species, we can also use it as a physical model to generate the next set of testable hypotheses that will lead to new discoveries in animal climbing.

\section{ACKNOWLEDGMENTS}

This work was supported in part by DARPA/SPAWAR Contract N66001-05-C-8025. Jonathan Clark is supported by the IC Postdoctoral Fellow Program under grant number HM158204-1-2030. In addition we would like to thank $\mathrm{Al}$ Rizzi, Martin Buehler, and Aaron Saunders for a number of helpful discussions bearing on design and control of the physical platform.

\section{REFERENCES}

[1] C. Balaguer, A. Gimenez, J. Pastor, V. Padron, and C. Abderrahim, "A climbing autonomous robot for inspection applications in $3 \mathrm{~d}$ complex environments." Robotica, vol. 18, pp. 287-297, 2000.

[2] G. La Rosa, M. Messina, G. Muscato, and R. Sinatra, "A low-cost lightweight climbing robot for the inspection of vertical surfaces." Mechatronics, vol. 12, no. 1, pp. 71-96, 2002.

[3] D. Bevly, S. Dubowsky, and C. Mavroidis, "A simplified cartesiancomputed torque controller for highly geared systems and its application to an experimental climbing robot." Transactions of the ASME. Journal of Dynamic Systems, Measurement and Control, vol. 122, no. 1, pp. 27-32, 2000.

[4] T. Bretl, S. Rock, and J. C. Latombe, "Motion planning for a threelimbed climbing robot in vertical natural terrain," in IEEE International Conference on Robotic and Automotion (ICRA 2003), vol. 205, Taipei, Taiwan, 2003, pp. 2946 - 295.

[5] K. Autumn, M. Buehler, M. R. Cutkosky, R. Fearing, R. Full, D. Goldman, R. Groff, W. Provancher, A. Rizzi, U. Saranli, A. Saunders, and D. Koditschek, "Robotics in scansorial environments," in Unmanned Systems Technology VII, D. W. G. G. R. Gerhart, C. M. Shoemaker, Ed., vol. 5804. SPIE, 2005, pp. 291-302.

[6] "http://www.vortexhc.com/vmrp.html."
[7] J. Xiao, A. Sadegh, M. Elliot, A. Calle, A. Persad, and H. M. Chiu, "Design of mobile robots with wall climbing capability," in Proceedings of IEEE AIM, Monterey, CA, Jul. 24-28, 2005, pp. 438-443.

[8] D. K.A., H. A.D., G. S., R. R.E., and Q. R.D., "A small wall-walking robot with compliant, adhesive feet," in Proceedings of IROS, Edmonton, Canada.

[9] C. Menon, M. Murphy, and M. Sitti, "Gecko inspired surface climbing robots," in Proceedings of IEEE ROBIO, Aug. 22-26, 2004, pp. 431-436.

[10] “http://www.irobot.com."

[11] D. I. Goldman, T. S. Chen, D. M. Dudek, and R. J. Full, "Dynamics of rapid vertical climbing in a cockroach reveals a template," Journal of Experimental Biology, vol. 209, pp. 2990-3000, 2006.

[12] R. J. Full and D. E. Koditschek, "Templates and anchors: Neuromechanical hypotheses of legged locomotion on land,' Journal of Experimental Biology, vol. 202, no. 23, pp. 3325-3332, 1999.

[13] R. Blickhan and R. J. Full, "Similarity in multilegged locomotion: Bounding like a monopod," Journal of Comparative Physiology, vol. 173, no. 5, pp. 509-517, 1993.

[14] G. A. Cavagna, N. C. Heglund, and T. C. R., "Mechanical work in terrestrial locomotion: Two basic mechanisms for minimizing energy expenditure," American Journal of Physiology, vol. 233, 1977.

[15] T. A. MCMahon and G. C. Cheng, "Mechanics of running. how does stiffness couple with speed?" Journal of Biomechanics, vol. 23, no. 1, pp. 65-78, 1990.

[16] J. Schmitt and P. Holmes, "Mechanical models for insect locomotion: Dynamics and stability in the horizontal plane i. theory," Biological Cybernetics, vol. 83, no. 6, pp. 501-515, 2000.

[17] J. E. Clark, D. I. Goldman, T. S. Chen, R. J. Full, and D. Koditschek, "Toward a dynamic vertical climbing robot," in International Conference on Climbing and Walking Robots (CLAWAR). Brussels, Belgium: Professional Engineering Publishing, 2006, vol. 9.

[18] K. Autumn, S. T. Hsieh, D. M. Dudek, J. Chen, C. Chitaphan, and R. J. Full, "Dynamics of geckos running vertically," Journal of Experimental Biology, vol. 209, pp. 260-270, 2006.

[19] L. H. Ting, R. Blickhan, and R. J. Full, "Dynamic and static stability in hexapedal runners," Journal of Experimental Biology, vol. 197, pp. 251-269, 1994.

[20] J. Schmitt, M. Garcia, R. Razo, P. Holmes, and R. Full, "Dynamics and stability of legged locomotion in the horizontal plane: a test case using insects." Biological Cybernetics, vol. 86, no. 5, pp. 343-53, 2002.

[21] M. Spenko, M. R. Cutkosky, R. Majidi, R. Fearing, R. Groff, and K. Autumn, "Foot design and integration for bioinspired climbing robots," in In review for Unmanned Systems Technology VIII. SPIE, 2006.

[22] R. M. Alexander and A. S. Jayes, "A dynamic similarity hypothesis for the gaits of quadrupedal mammals," Journal of Zoology, vol. 201, pp. 135-152, 1983.

[23] R. M. Alexander, Principles of Animal Locomotion. Princeton University Press, 2003.

[24] U. Saranli, M. Buehler, and D. E. Koditschek, "Rhex: A simple and highly mobile hexapod robot," International Journal of Robotics Research, vol. 20, no. 7, pp. 616-631, 2001.

[25] G. C. Haynes and A. Rizzi, "Gait regulation and feedback on a robotic climbing hexapod," in Proceedings of Robotics: Science and Systems, August 2006.

[26] E. Klavins, H. Komsuoglu, R. J. Full, and D. E. Koditschek, The Role of Reflexes Versus Central Pattern Generators in Dynamical Legged Locomotion. MIT Press, 2002, ch. Neurotechnology for Biomimetic Robots, pp. 351-382.

[27] E. Klavins and D. E. Koditschek, "Phase regulation of decentralized cyclic robotic systems," International Journal of Robotics Reserach, vol. 21, no. 3, pp. 257-275, 2002.

[28] M. Buehler, D. E. Koditschek, and P. J. Kindlmann, "Planning and control of a juggling robot," International Journal of Robotics Research, vol. 13, no. 2, pp. 101-118, 1994.

[29] A. A. Rizzi and D. E. Koditschek, "An active visual estimator for dexterous manipulation,' IEEE Transactions on Robotics and Automation, vol. 12 , no. 5, pp. 697-713, 1996.

[30] M. Buehler, D. E. Koditschek, and P. J. Kindlmann, "A simple juggling robot: Theory and experimentation," in Experimental Robotics I. Spring-Verlag, 1990, pp. 35-73. 